\title{
Diabetic Ketoacidosis Due to Type 1 Diabetes Mellitus Induced by Interferon Therapy for Chronic Hepatitis C During the Course of Hashimoto's Thyroiditis: Case Report
}

\author{
Rie Jo ${ }^{1,2 *}$, Kyoko Iwase ${ }^{1}$, Toshihiro Nishizawa ${ }^{3}$ and Hisaji Ohshima ${ }^{1}$
}

${ }^{1}$ Department of Internal Medicine, National Hospital Organization Tokyo Medical Center, Tokyo, Japan

${ }^{2}$ Department of Internal Medicine, Keiyu Hospital, Kanagawa, Japan

${ }^{3}$ Department of Gastroenterology, National Hospital Organization Tokyo Medical Center, Tokyo, Japan

\begin{abstract}
Interferon a (IFNa) has been reported to induce several autoimmune diseases, including autoimmune thyroiditis (AITD) and type 1 diabetes (T1D). In addition, co-occurrence of T1D and AITD is well known as one of the variants of the autoimmune polyglandular syndrome type 3. Meanwhile, there are no reports about the risk of development of IFN treatment-related T1D in patients with previously diagnosed AITD. We present a single case report about a 61-yearold Japanese woman with Diabetic Ketoacidosis (DKA) three months after the initiation of the combination therapy with peginterferon $a-2 b$ and ribavirin for chronic hepatitis $C$. She was diagnosed with T1D owing to severely impaired insulin secretion along with DKA and presence of positive GAD antibody. Her T1D was supposed to be induced by IFN therapy. She had received the replacement therapy for hypothyroidism resulting from Hashimoto's thyroiditis for 30 years. The patient had human leukocyte antigens (HLA), DRB1*04:05 and DQB1*04:01, which are associated with a genetic predisposition for T1D and AITD. Patients with previously diagnosed AITD are assumed to be at high risk for IFN treatment-related T1D because most patients with T1D triggered by IFN were reported to have genetic susceptibilities similar to those with classical T1D and AITD. In conclusion, patients with known AITD should be carefully monitored for their glycemic condition to avoid life-threatening events such as DKA when they receive IFN therapy.
\end{abstract}

Keywords: Interferon; Type 1 diabetes; Diabetic ketoacidosis; Hashimoto's thyroiditis; Chronic hepatitis C

\section{Introduction}

Interferons (IFNs) are cytokines induced by viral infection or other immune triggers. They are currently utilized in the treatment of chronic viral hepatitis and several cancers (e.g., renal cell carcinoma and melanoma) because of their antiviral and antitumor effects. IFNa, which increases expression of the Major Histocompatibility Complex (MHC) class I antigen and activities of T cells and natural killer cells [1], causes some autoimmune diseases including autoimmune thyroid disease (AITD) and type 1 diabetes (T1D) [2]. In addition, co-occurrence of T1D and AITD is well known as one of the variants of the autoimmune polyglandular syndrome type 3 (APS 3 variant). We describe the case of a patient with diabetic ketoacidosis (DKA) due to T1D induced by peginterferon $a-2 b$ (PEG-IFNa-2b) and ribavirin (RBV) therapy for chronic hepatitis $\mathrm{C}$ during the course of Hashimoto's thyroiditis. There are no reports about the risk of development of IFN treatment-related T1D in patients with previously diagnosed AITD. We report the case and discuss the association between IFN treatment-related T1D and AITD.

\section{Case Report}

A 61-year-old woman with progressive nausea and appetite loss visited our hospital. She is a Japanese dressmaker. At the age of 30 years, she was simultaneously diagnosed with chronic hepatitis $\mathrm{C}$ in addition to hypothyroidism resulting from Hashimoto's thyroiditis. Her thyroid function was maintained with levothyroxine administered at a dose of $50 \mu \mathrm{g} /$ day to $75 \mu \mathrm{g} /$ day. At the age of 60 years, combination therapy with PEG-IFNa-2b ( $80 \mu \mathrm{g} /$ week) and RBV $(600 \mathrm{mg} /$ day $)$ was initiated because her liver function became exacerbated, as indicated by an AST level of $252 \mathrm{IU} / \mathrm{L}$. Her level of $\mathrm{HbA} 1 \mathrm{c}$ was $6.3 \%$ at the initiation of the combination therapy and she had never been diagnosed with diabetes mellitus. Two months after initiating therapy with PEG-IFNa-2b plus $\mathrm{RBV}$, she experienced weight loss (10 kg in a month) and suffered from thirst and polyuria. With a 4-week history of these symptoms, she visited our hospital because of worsening nausea and appetite loss, and was admitted on an urgent basis. On admission, her body weight was 44 $\mathrm{kg}$, body mass index was $16.0 \mathrm{~kg} / \mathrm{m}^{2}$, consciousness level was E4V5M6 (Glasgow Coma Scale), blood pressure was $132 / 106 \mathrm{mmHg}$, pulse rate was $121 /$ minute, and respiratory rate was $23 /$ minute with Kussmaul respiration. On physical examination, no remarkable findings were observed, except dryness of the mouth. Laboratory results revealed hyperglycemia (blood glucose, $551 \mathrm{mg} / \mathrm{dL}$; HbAlc, 8.3\%; and glycoalbumin, $48.2 \%)$. Urine ketone was strongly positive (3+). Venous blood gas analysis revealed metabolic acidosis with increased anion gap ( $\mathrm{pH}, 7.238 ; \mathrm{PCO}_{2}, 27.6 \mathrm{mmHg} ; \mathrm{HCO}_{3}^{-}, 11.5 \mathrm{mmol} / \mathrm{L}$; base excess, -14.4 $\mathrm{mmol} / \mathrm{L}$; and anion gap, $17.5 \mathrm{mmol} / \mathrm{L}$ ). Her renal function was normal (blood urea nitrogen, $22.1 \mathrm{mg} / \mathrm{dL}$; creatinine, $0.67 \mathrm{mg} / \mathrm{dL}$ ). High blood glucose accompanied by metabolic acidosis and urine ketone led to the diagnosis of DKA. The level of anti-glutamic acid decarboxylase (GAD) antibody was markedly high $(2000 \mathrm{U} / \mathrm{mL}$, determined by radioimmunoassay). Her insulin secretion was severely impaired; serum C peptide was $0.05 \mathrm{ng} / \mathrm{mL}$ during fasting and $0.08 \mathrm{ng} / \mathrm{mL}$ at 2 hours post-prandium, and urinary excretion of C peptide was $1.61 \mu \mathrm{g} /$ day. She was diagnosed with T1D owing to severely impaired insulin secretion along with DKA and presence of positive GAD antibody. Her

*Corresponding author: Rie Jo. Department of Internal Medicine, Keiyu Hospital, 3-7-3 Minatomirai, Nishi-ku, Yokohama, Kanagawa, 220-8521, Japan; Tel: +81-45221-8181; E-mail: r.oga925@nifty.com

Received: June 14, 2017; Accepted: July 04, 2017; Published: July 09, 2017

Citation: Jo R, Iwase K, Nishizawa T, Ohshima H (2017) Diabetic Ketoacidosis Due to Type 1 Diabetes Mellitus Induced by Interferon Therapy for Chronic Hepatitis C During the Course of Hashimoto's Thyroiditis: Case Report. Diabetes Case Rep 2 126. doi: $10.4172 / 2572-5629.1000126$

Copyright: @ 2017 Jo R, et al. This is an open-access article distributed unde the terms of the Creative Commons Attribution License, which permits unrestricted use, distribution, and reproduction in any medium, provided the original author and source are credited. 


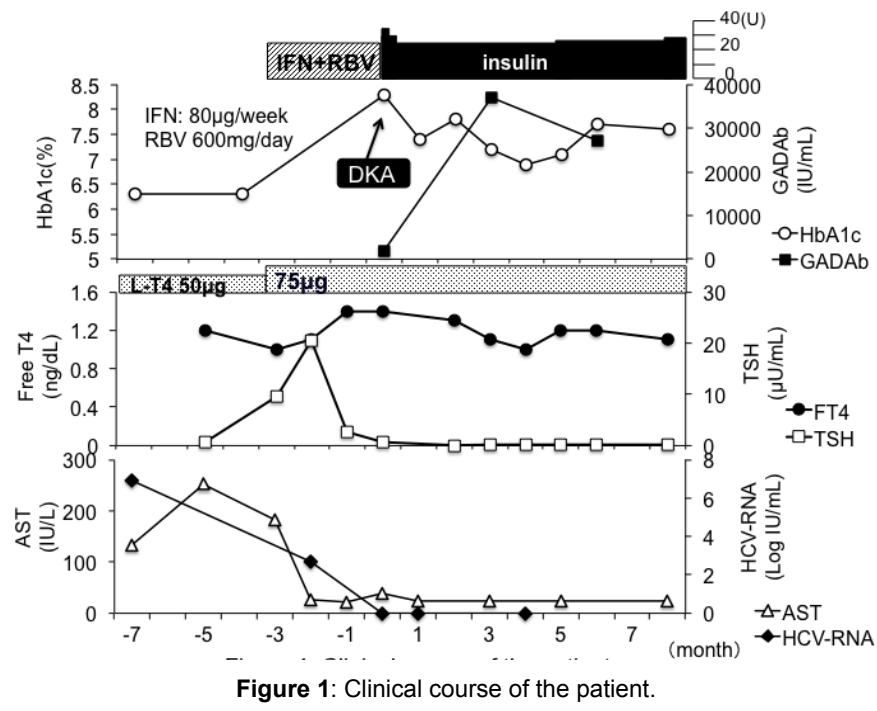

human leukocyte antigen (HLA) types included DRB1*04:05/14:05 and DQB1*04:01:01/05:03:01, which indicate high susceptibility to T1D. Although serum lipase (78 IU/L) and elastase-1 (302 ng/dL) levels were elevated on admission, symptoms suggestive of acute pancreatitis were not observed. Abdominal ultrasonography revealed chronic liver damage (a dulled edge of the right hepatic lobe), and liver cirrhosis was ruled out. She had no complications associated with diabetes.

The clinical course is summarized in Figure 1. On admission, continuous insulin injection (two to four units/hour) and fluid administration were started. Her blood gas was normalized on the second day. Subsequently, basal-bolus insulin therapy was initiated. At the time of discharge, she was administered insulin glargine, 5 units/ day and insulin aspart, 20 units/day. The titer of GAD antibody was elevated to $39,000 \mathrm{IU} / \mathrm{mL}$ at three months after discharge and then peaked (Figure 1). Her HbAlc remained around $7.5 \%$ with 25 units to 30 units of insulin per day after discharge.

Her antithyroglobulin antibody level was elevated $(47 \mathrm{IU} / \mathrm{mL})$ on admission, which was compatible with Hashimoto's thyroiditis. Her thyroid function had been maintained with $75 \mu \mathrm{g}$ of levothyroxine at the initiation of PEG-IFNa-2b and RBV therapy. However, her TSH level was transiently elevated to $20 \mu \mathrm{U} / \mathrm{mL}$ one month after the initiation of combination therapy and then spontaneously normalized (Figure 1). Since then, her thyroid function has been normal (TSH, $0.66 \mu \mathrm{L} / \mathrm{mL}$ and free $\mathrm{T} 4,1.4 \mathrm{ng} / \mathrm{dL}$ ) with levothyroxine $75 \mu \mathrm{g}$.

PEG-IFN $a-2 b$ and RBV were discontinued on admission because they were suspected of inducing T1D. Although IFN-RBV therapy was not completely accomplished, the patient achieved a sustained virological response. She remained negative for hepatitis $\mathrm{C}$ virus (HCV) RNA for two years since discontinuation of the therapy (Figure 1).

\section{Discussion}

The case at presentation partly fulfilled the diagnostic criteria of fulminant T1D, with $\mathrm{HbA} 1 \mathrm{c}$ level $<8.7 \%$, urinary C-peptide excretion rate $<10 \mu \mathrm{g} /$ day, and fasting serum C-peptide level $<0.3 \mathrm{ng} / \mathrm{mL}$ and $<0.5 \mathrm{ng} / \mathrm{mL}$ post-prandium [3]. However, the duration from the onset of hyperglycemic symptoms to occurrence of DKA was about 4 weeks, which did not meet the criterion of fulminant T1D. Isletrelated autoantibodies, such as antibodies against GAD, islet-associated antigen 2, and insulin, are generally undetectable in fulminant T1D [3].
However, the titer of GAD antibody was markedly high in this case. Therefore, the presenting case would be diagnosed as acute-onset T1D [4] based on a high titer of GAD antibody and the time taken for onset of symptoms. Although it is unknown when GAD antibody converted to be positive in this case, the patient is hypothesized to newly develop T1D induced by IFNa, considering the clinical course.

IFNa is known to increase MHC class I antigen expression and activities of T cells and natural killer cells [1]. Although the pathogenesis of IFN treatment-related T1D has not been clearly elucidated, an association between IFNa and T1D has been reported. Huang et al. [5] reported that IFNa was significantly overexpressed in the pancreases of patients with T1D. Stewart et al. [6] reported that transgenic mice in which $\beta$ cells overexpressed IFN $\alpha$ developed hypoinsulinemic diabetes, and a neutralizing antibody against IFNa prevented its development.

The rate of incidence of IFN treatment-related T1D in Japan is reported to be $0.7 \%$ to $0.96 \%$ [2]. Further, PEG-IFN and RBV combination therapy shortens the time period from the initiation of IFN therapy to the onset of T1D compared with single therapy with nonpegylated IFN [7]. The results of a nationwide survey of IFN treatment-related T1D in Japan were reported in 2011 [7]. In this survey, HLA-DR4 (34.6\% of total subjects) and DR9 (26.9\% of total subjects), which are known as classical T1D susceptibility HLA-DRs in the Japanese population [8], were associated with IFN treatmentrelated T1D [7]. In addition, the prevalence of HLA-DR13 (15.4\% of total subjects) was specifically higher in patients with IFN treatmentrelated T1D than in healthy control subjects and patients with classical T1D [7]. The results of the nationwide survey in Japan [7] indicated that patients with HLA-DR4 or HLA-DR9, who are naturally susceptible to T1D, are likely to develop T1D triggered by IFN therapy. In addition, IFN-associated T1D is characterized by a higher level of GAD antibodies [9], which applies equally to the present case whose levels of GAD antibodies were $2000 \mathrm{IU} / \mathrm{mL}$ on admission and $39,000 \mathrm{IU} / \mathrm{mL}$ at 3 months after diagnosis.

IFN therapy has also been noted to trigger AITD. Some studies have reported that $1 \%$ to $40 \%$ of IFN-treated patients developed positive thyroid autoantibodies, and $3.4 \%$ to $31.3 \%$ developed AITD during IFN therapy [10]. Therefore, thyroid function should be closely monitored during IFN therapy. Possible mechanisms for development of IFN- induced thyroiditis include MHC class I upregulation, Th1 
Citation: Jo R, Iwase K, Nishizawa T, Ohshima H (2017) Diabetic Ketoacidosis Due to Type 1 Diabetes Mellitus Induced by Interferon Therapy for Chronic Hepatitis C During the Course of Hashimoto's Thyroiditis: Case Report. Diabetes Case Rep 2: 126. doi: 10.4172/2572-5629.1000126

Page 3 of 3

switching and direct effects on the thyroid [10]. There are some reports of co-occurrence of T1D and AITD in patients receiving IFN therapy [11]. In the nationwide survey in Japan described above, cooccurrence of T1D and AITD was observed in $27.5 \%$ of patients with IFN treatment-related T1D [7]. However, the rate of patients who had previously diagnosed with AITD before initiation of IFN therapy was not reported in this survey. The patient in our study was characterized by precedence of overt hypothyroidism before initiation of IFN therapy. In addition, TSH was transiently elevated immediately after initiation of IFN therapy (Figure 1). Although the etiology of the transient elevation of TSH remained unclear, there are two possibilities. One is that transient exacerbation of AITD may have occurred due to modulation of thyroid autoimmunity by IFN. The other is that thyroid function may have suppressed by de novo thyroiditis directly induced by IFN as Oppenheim et al. [10] reported. In case of the former, the titers of thyroid autoantibodies would have changed; however, we were not able to compare those of before and after initiation of IFN therapy.

The co-occurrence of T1D and AITD is frequently observed, which is one of the variants of APS 3. The frequency of positive thyroid antibodies in T1D has been reported as $8 \%$ to $44 \%$ [12], and $16 \%$ to $50 \%$ of these patients progress to clinical AITD [13]. The association between T1D and AITD is most likely due to shared genetic predisposition [13]. Several loci and genes that are involved in immune regulation have been shown to contribute to the joint susceptibility to T1D and AITD [13]. HLA class II genes play a major role in the joint susceptibility to T1D and AITD. DR3 and DR4, especially DR3-DQB1 ${ }^{\star} 02: 01$ and DR4-DQB1 ${ }^{\star} 03: 02$, have been reported to be associated with the combined phenotype of T1D and AITD in Caucasians. In addition, the study in Japan has shown the association of HLA-DRB1 ${ }^{\star} 08: 02$ DQA $1^{\star}$ 04:01-DQB1 ${ }^{\star} 04: 02, \quad \mathrm{DRB}^{*}{ }^{\star} 04: 05-\mathrm{DQA} 1^{\star} 03: 03-\mathrm{DQB} 1^{\star} 04: 01$ and DRB1 ${ }^{\star} 08: 02-\mathrm{DQA} 1^{\star} 03: 01-\mathrm{DQB1} 1^{\star} 03: 02$ with T1D and AITD [14]. In the presenting case, $\mathrm{DRB} 1^{*} 04: 05$ and $\mathrm{DQB} 1^{*} 04: 01$ might share a genetic predisposition for T1D and AITD.

To our knowledge, it remains to be elucidated whether T1D is more likely to be induced by IFNa in patients with previously diagnosed AITD. However, AITD is assumed to be a possible risk factor for IFN treatment-related T1D because the majority of patients with T1D triggered by IFN have genetic predispositions similar to those with classical T1D and AITD. In this case, transient suppression of thyroid function, which may have come from exacerbation of thyroiditis, and development of T1D were observed at nearly the same time. This phenomenon may have reflected the common immunological changes on thyroid and pancreas derived from IFN therapy.

\section{Conclusion}

We have described the case of a patient with Hashimoto's disease that subsequently developed T1D induced by IFN therapy. She had HLA-
$\mathrm{DRB1}{ }^{\star}$ 04:05 and DQB1 ${ }^{\star} 04: 01$ as a common genetic predisposition for T1D and AITD. Patients with AITD are assumed to be at high risk for IFN treatment-related T1D because the majority of patients with T1D triggered by IFNa have genetic predispositions similar to those with classical T1D and AITD. Therefore, patients with known AITD should be closely monitored for their glycemic condition during IFN therapy to avoid life-threatening events such as DKA.

\section{References}

1. Tilg $H$ (1997) New insights into the mechanisms of interferon alfa: An immunoregulatory and anti-inflammatory cytokine. Gastroenterology 112: 1017-1021.

2. Okanoue T, Sakamoto S, Itoh Y, Minami M, Yasui K, et al. (1996) Side effects of high-dose interferon therapy for chronic hepatitis C. J Hepatol 25: 283-291.

3. Imagawa A, Hanafusa T, Awata T, Ikegami H, Uchigata Y, et al. (2012) Report of the committee of the Japan Diabetes Society on the Research of fulminant and acute-onset type 1 diabetes mellitus: New diagnostic criteria of fulminant type 1 diabetes mellitus. J Diabetes Investig 3: 536-539.

4. Kawasaki E, Maruyama T, Imagawa A, Awata T, Ikegami H, et al. (2014) Diagnostic criteria for acute-onset type 1 diabetes mellitus (2012): Report of the Committee of Japan Diabetes Society on the Research of fulminant and acute-onset type 1 diabetes mellitus. J Diabetes Investig 5: 115-118.

5. Huang X, Yuang J, Goddard A, Foulis A, James R F, et al. (1995) Interferon expression in the pancrea of patients with type I diabetes. Diabetes 44 658-664.

6. Stewart TA, Hultgren B, Huang X, Pitts-Meek S, Hully J, et al. (1993) Induction of type I diabetes by interferon-alpha in transgenic mice. Science 260: $1942-1946$

7. Nakamura K, Kawasaki E, Imagawa A, Awata T, Ikegami H, et al. (2011) Type 1 diabetes and interferon therapy: A nationwide survey in Japan. Diabetes Care 34: 2084-2089.

8. Kawasaki E, Matsuura N, Eguchi K (2006) Type 1 diabetes in Japan Diabetologia 49: 828-836.

9. Nakanishi K, Saitoh S (2011) Clinical and genetic characteristics of patients with type 1 diabetes associated with interferon therapy. Diabetes Care 34 471-473.

10. Oppenheim Y, Ban Y, Tomer Y (2004) Interferon induced Autoimmune Thyroid Disease (AITD): A model for human autoimmunity. Autoimmun Rev 3: 388-393.

11. Kose S, Gozaydin A, Akkoclu G, Ece G (2012) Chronic hepatitis B with type I diabetes mellitus and autoimmune thyroiditis development during interferon alpha therapy. J Infect Dev Ctries 6: 364-368.

12. Kordonouri O, Klinghammer A, Lang EB, Gruters-Kieslich A, Grabert M, et al. (2002) Thyroid autoimmunity in children and adolescents with type 1 diabetes: A multicenter survey. Diabetes Care 25: 1346-1350.

13. Huber A, Menconi F, Corathers S, Jacobson EM, Tomer Y (2008) Joint genetic susceptibility to type 1 diabetes and autoimmune thyroiditis: From epidemiology to mechanisms. Endocr Rev 29: 697-725.

14. Hashimoto K, Maruyama H, Nishiyama M, Asaba K, Ikeda $\mathrm{Y}$, et al. (2005) Susceptibility alleles and haplotypes of human leukocyte antigen DRB1 DQA1, and DQB1 in autoimmune polyglandular syndrome type III in Japanese population. Horm Res 64: 253-260. 